\title{
Frutos, semillas, y mamíferos frugívoros: diversidad funcional de interacciones poco estudiadas
}

\author{
J.M. Fedriani ${ }^{1,2, *}$, A. Suárez-Esteban ${ }^{3,4}$ \\ (1) Centro de Ecologia Aplicada Prof. Baeta Neves/InBIO, Instituto Superior de Agronomia, Universidade de Lisboa, Tapada da Ajuda, $1349-017$ Lisboa, \\ Portugal. \\ (2) Estación Biológica de Doñana (EBD-CSIC). Américo Vespucio s/n 41092, Sevilla, España. \\ (3) Department of Renewable Resources. University of Alberta. 116 St. and 85 Ave., Edmonton, AB, Canada. T6G 2R3. \\ (4) Yukon College. 500 College Drive, Whitehorse, YT, Canada. Y1A 5D4.
}

.*Autor de correspondencia: J.M. Fedriani [fedriani@ebd.csic.es]

> Recibido el 20 de diciembre de 2015 - Aceptado el 20 de diciembre de 2015

Fedriani, J.M., Suárez-Esteban, A. 2015. Frutos, semillas, y mamíferos frugívoros: diversidad funcional de interacciones poco estudiadas.

Ecosistemas 24(3): 1-4. Doi.: 10.7818/ECOS.2015.24-3.01

Las interacciones entre frutos, semillas, y sus consumidores son de gran importancia para el funcionamiento y conservación de casi todos los ecosistemas terrestres (Levey et al. 2002, Bronstein 2007, Fleming y Krebbs 2013, Bascompte y Jordano 2014). Por una parte, las plantas producen distintos tipos de frutos (carnosos, legumbres, secos) que proporcionan nutrientes, en muchos casos críticos, para la supervivencia y reproducción de vertebrados, invertebrados y microorganismos. Por otra parte, dichos consumidores pueden ejercer una miríada de efectos positivos y negativos, directos e indirectos, ecológicos y evolutivos, sobre las poblaciones y comunidades vegetales de las que se alimentan. La diversidad e importancia funcional caracterizan a todos los grupos de consumidores mencionados, siendo los mamíferos frugívoros un ejemplo paradigmático que indudablemente merece particular atención.

Numerosos órdenes de mamíferos terrestres incluyen especies consumidoras de frutos y semillas de muchas especies vegetales, siendo esto cierto no solo en el caso de especies típicamente frugívoras (primates, muchos quirópteros), granívoras (roedores) o herbívoras (ungulados, lagomorfos) sino, también, para muchos miembros del orden Carnivora (principalmente mustélidos, cánidos y úrsidos; Willson 1993, Fedriani 1996, Traveset y Willson 1997, Herrera 2002). Además, estas interacciones también están ampliamente representadas en diversos ecosistemas subárticos, boreales, templados, mediterráneos, tropicales, y áridos (Levey et al. 2002, Bronstein 2007, Terborgh 2010, Fleming y Krebbs 2013). Así mismo, las interacciones entre mamíferos y frutos ocurren tanto en ambientes humanizados como en aquellos bien preservados (p.ej. Fedriani et al. 2001). Es llamativo, sin embargo, el hecho de que en ambientes templados y mediterráneos, habitualmente muy humanizados, las interacciones planta-mamífero frugívoro han sido relativamente poco estudias, en comparación tanto al importante esfuerzo de investigación en estos ambientes sobre frugivoría y dispersión de semillas por aves (Herrera et al. 1994, Jordano y Schupp 2000, Rey y Alcántara 2000, García et al. 2005, entre otros muchos), como en relación a los numerosos estudios sobre mamíferos frugívoros (primates, tapires, roedores) en ambientes tropica- les (p.ej. Fragoso 1997, Chapman y Russo 2006, Fleming y Krebbs 2013). La dispersión y depredación de bellotas del género Quercus por roedores fueron previamente revisadas (véase Pérez-Ramos et al. 2014) por lo que no son nuevamente tratadas aquí. Sin embargo, sí resulta oportuno considerar las interacciones entre plantas y otros mamíferos consumidores de frutos y semillas, con particular énfasis en los ambientes humanizados.

En este monográfico comprende seis trabajos, tres de investigación y otros tres de revisión. De los tres trabajos de investigación, uno de ellos identifica interacciones mutualistas y competitivas en un sistema formado por frutos de cítricos, mamíferos frugívoros, y hongos (Peris et al. 2015) y los otros dos presentan el papel de mamíferos domésticos como dispersores de semillas en ambientes mediterráneos (Macilla-Leytón et al. 2015, Ramos-Font et al. 2015). En cuanto a los tres trabajos de revisión, dos de ellos repasan de forma detallada la importancia de los mamíferos silvestres en la (re)colonización de hábitat naturales y humanizados (EscribanoÁvila et al. 2015, González-Varo et al. 2015), y el tercer ejemplariza la complejidad de este tipo de sistemas ecológicos y la conveniencia de una aproximación integradora para su estudio (Campos y Velez 2015). Por tanto, el presente monográfico ilustra la diversidad funcional y de hábitats que caracteriza las interacciones entre plantas y mamífero frugívoros y, por otra parte, facilita la identificación de algunos aspectos menos estudiados. Con ello, pretendemos estimular futuras líneas de investigación y enfatizar la importancia de este tipo de interacciones para el funcionamiento y conservación de los ecosistemas, tanto naturales como humanizados.

\section{Dispersión de semillas por mamíferos en ambientes humanizados}

Los ambientes humanizados presentan una serie de características relevantes en relación a las interacciones entre plantas y frugívoros. En primer lugar, la actividad humana (fragmentación, cambios en el uso del suelo, caza) conlleva un empobrecimiento general de la biodiversidad y, en concreto, de vertebrados frugívoros de mayor tamaño (algunas aves y mamíferos). Este proceso de "defaunación selectiva" (Dirzo et al. 2014) afecta intensamente a 
muchos mamíferos frugívoros de mayor tamaño que son más intensamente explotados por la caza legal e ilegal, y puede conllevar que especies de plantas con mayor tamaño de frutos experimenten severa limitación de la dispersión de sus semillas (p.ej. Effiom et al. 2014). Así mismo, en estos ambientes son frecuentes las estructuras lineales (carreteras, caminos, cortafuegos) que, en algunos casos pueden actuar como barreras, y en otros pueden ser seleccionadas por mamíferos frugívoros como corredores (SuárezEsteban et al. 2013a). Con ello, estas estructuras facilitan la (re)coIonización de hábitats vacantes por especies vegetales nativas e invasoras (Padrón et al. 2011, Suárez-Esteban et al. 2013b, González-Varo et al. 2015), tal y como ocurre en los campos abandonados (Escribano-Ávila et al. 2015).

Los ambientes humanizados muestran con frecuencias escasa cobertura vegetal. A diferencia del patrón típico de las aves frugívoras, estos hábitats abiertos sí son usados con frecuencia por distintos mamíferos frugívoros (ungulados, lagomorfos, carnívoros) por lo que proveen de servicios de dispersión complementarios a los de las aves (Escribano-Ávila et al. 2015, González-Varo et al. 2015). Así mismo, muchos mamíferos como los carnívoros y ungulados muestran alta movilidad y largos tiempos de retención de las semillas ingeridas, por lo que dispersan las semillas a larga distancia, lo cual también facilita la recolonización de estos hábitats vacantes y el flujo génico entre poblaciones (Fedriani y Delibes 2011, Escribano-Ávila et al. 2015, González-Varo et al. 2015). No obstante, el patrón espacial de la lluvia de semillas generadas por los mamíferos tiende a ser fuertemente agregado a varias escalas espaciales (Fedriani y Wiegand 2014). Por ello, resultan ineludibles detalladas investigaciones que identifiquen los efectos poblacionales, probablemente negativos, de dicha agregación, así como los intrigantes y escasamente conocidos procesos post-dispersivos que podrían atenuarlos (p.ej. Enders y Vander Wall 2012).

Un caso particular de estos ambientes humanizados lo constituyen los cultivos y las zonas de pastoreo para el ganado doméstico. Muchas especies de mamíferos silvestres consumen frecuentemente frutos domésticos en sistemas agrícolas de todo el mundo (Fedriani et al. 2001, Rosalino y Santo-Reis 2009, González-Varo et al. 2015, Peris et al. 2015). Estos sistemas son particularmente apropiados para evaluar algunas cuestiones ecológicas, tal y como ejemplariza el estudio de Peris et al. (2015) donde se evalúa la naturaleza de las interacciones en la triada formada por cítricos, mamíferos frugívoros y hongos. Por otra parte, muchas especies de mamíferos domésticos también consumen frutos silvestres, contribuyendo de forma notable a su dispersión (ManciIla-Leyton et al. 2015, Ramos-Font et al. 2015). Por ejemplo, el ganado caprino favoreció la germinación y dispersión de semillas de tres especies de cistáceas (Mancilla-Leyton et al. 2015) y las ovejas pueden ser dispersantes eficaces de semillas de seis especies de leguminosas (Ramos-Font et al. 2015).

\section{Interacciones planta-mamífero frugívoro: más allá de la dispersión de semillas}

Las interacciones entre especies están caracterizadas por costes y beneficios que son altamente cambiantes en el espacio y el tiempo (i.e. contexto-dependientes; Bronstein 1994, Holland y DeAngelis 2010), y las interacciones plantas-mamífero frugívoro no son una excepción al respecto (p.ej. Fedriani y Delibes 2011, Fedriani et al. 2012, Campos y Velez 2015). Sin embargo, en el estudio de las interacciones entre plantas y mamíferos frugívoros existe cierta tendencia a enfatizar los posibles beneficios (p.ej. mayores tasas de germinación, llegada a hábitats vacantes, incremento del flujo génico) y a desconocer los inevitables costes asociados. Un interesante ejemplo de los costes asociados a estas interacciones es el estudio de Mancilla-Leyton et al. (2015), donde el ganado caprino actuó fundamentalmente cómo depredador de semillas de arbustos de frutos carnosos, aunque llegó a dispersar algunas semillas. Por su parte, Ramos-Font et al. (2015) documentan como el estiércol de ganado ovino puede dificultar la germinación de semillas y emergencia de plántulas de varias especies de legumino- sas silvestres (véase también Campos y Velez 2015). Por tanto, existe un continuo de resultados en relación a las interacciones entre plantas y mamíferos frugívoros, que van desde el antagonismo al mutualismo, dependiendo de la identidad de las especies que interactúan y del contexto ecológico en el que tienen lugar (p.ej. Perea et al. 2013). Un entendimiento completo de las interacciones planta-mamífero en particular, y de las interacciones entre especies en general, requiere la consideración simultánea de sus costes y beneficios a distintos niveles.

Con frecuencia los ecólogos ejercemos nuestra labor condicionados por prejuicios y estereotipos que pueden limitar el alcance de nuestras investigaciones. Términos tales como "dispersante legítimo" o "dispersión dirigida" pueden sobresimplificar la complejidad de muchos procesos ecológicos y evolutivos que es, precisamente, lo que queremos entender. Muestra de ello son algunos mamíferos frugívoros que, en sus interacciones con las plantas en fruto, presentan comportamientos poco ortodoxos, no actuando ni como dispersantes ni como depredadores de semillas. En el matorral del Parque Nacional de Doñana (Huelva), Fedriani y Delibes $(2011,2013)$ encontraron que los conejos de campo Oryctolagus cunniculus consumen la pulpa de los frutos maduros, pero no las semillas, de piruétanos, Pyrus bourgaeana, y palmitos, Chamaerops humilis. Este tipo de frugívoros, que consumen la pulpa de los frutos pero no dispersa las semillas, son atrevidamente etiquetados como "ladrones" de pulpa, pues aparentemente no aportan beneficio alguno a las plantas con las que interactúan. Sin embargo, minuciosos experimentos de campo mostraron como el consumo de la pulpa por los conejos tuvo consecuencias demográficas inesperadas, y muy relevantes, para la dinámica de las poblaciones vegetales estudiadas. Por un lado, los frutos despulpados resultaron menos atractivos para algunos ungulados que habitualmente depredan sus semillas (i.e. beneficio de la interacción). Por otro lado, como la pulpa de los frutos supone una barrera física y química para algunos depredadores de semillas, los conejos despulpadores facilitaron la depredación de semillas por ratones e invertebrados (i.e. coste de la interacción; Fedriani y Delibes 2011, 2013, Rodríguez et al. 2014). El efecto neto para la planta de su interacción con los despulpadores es, por tanto, dependiente de las abundancias de otros frugívoros. Estas y otras interacciones heterodoxas entre plantas y animales son, probablemente, más frecuentes de lo que actualmente percibimos y constituyen una prometedora línea de investigación.

En el contexto de la importancia creciente de la teoría de la comunicación en investigaciones sobre la interacciones entre especies (p.ej. Raguso 2008, Schaefer y Duxon 2011, Rodríguez et al. 2013), la comunicación fruto-mamífero frugívoro mediada por los compuestos volátiles de los frutos supone una nueva avenida de investigación (p.e. Rodríguez et al. 2013, Peris et al. 2015, Whitehead et al. 2015). De forma análoga al caso de las interacciones planta-herbívoro (Freeland y Janzen 1974, Coley y Barone 1996, O'Reilly-Wapstra et al. 2004), es necesaria aun una identificación y cuantificación precisa de las presiones selectivas recíprocas entre plantas y mamíferos frugívoros en relación a la presencia y concentración de dichos compuestos (Cipollini y Levey 1997, Rodríguez et al. 2013, Whitehead et al. 2015). Por otra parte, desde una perspectiva de ecología animal, necesitamos también conocer mejor los mecanismos comportamentales y fisiológicos usados por los mamíferos para eludir y/o detoxificar los compuestos secundarios tóxicos de las plantas (Dearing et al. 2005). Por ejemplo, Fedriani y Boulay (2006) encontraron que los ratones de campo (Apodemus sylvaticus) en la Sierra de Cazorla (Jaén) utilizaban un sencillo mecanismo para 'manipular' las toxinas (i.e. glicósidos cardiacos) de la hierba perenne Helleborus foetidus. En concreto, revelaron que los ratones tendían a consumir in situ los frutos maduros y menos tóxicos de $\mathrm{H}$. foetidus, mientras que en el caso de los frutos verdes altamente tóxicos los almacenaban durante 1-3 días, permitiendo así la eliminación de gran parte de dichos compuestos tóxicos altamente volátiles (véase también Dearing 1997). Una atractiva posibilidad a tener en cuenta 
en futuros estudios es que tales mecanismos comportamentales y fisiológicos difieran en identidad y magnitud entre grupos funcionales de mamíferos (p.ej. depredadores versus dispersores de semillas; Samuni-Blank et al. 2013).

Así mismo, el papel que juegan las interacciones indirectas y las cascadas tróficas en las interacciones entre plantas y mamíferos frugívoros necesita ser investigado en mayor profundidad (Terborgh 2010, Fedriani y Delibes 2013, Escribano-Ávila et al. 2015). Por ejemplo, la presencia de depredadores tope como el lince ibérico (Lynx pardinus) probablemente alterare la abundancia y comportamiento no solo de dispersores de semillas como el zorro rojo (Vulpes vulpes; Fedriani et al. 1999), sino también de roedores y lagomorfos, cuyas funciones ecológicas pueden ser muy variables, tal y como señalamos arriba. Este tipo de sistemas, hasta la fecha escasamente considerado (Howe 1979, Fedriani y Boulay 2006), pueden abordarse satisfactoriamente combinando experimentos de campo bien replicados con modelos espacialmente explícitos basados en el individuo, que permiten, entre otras cosas, simular escenarios con distintas densidades de depredadores, mamíferos frugívoros y plantas en fruto (p.ej. Fedriani et al. 2015).

Por último, nos gustaría resaltar que para entender de forma exhaustiva el efecto de los mamíferos frugívoros sobre la dinámica y distribución de las plantas es necesario una aproximación integradora, donde se investiguen también los efectos de otros consumidores y especies que interactúan como, por ejemplo, insectos polinizadores (p.ej. Suárez-Esteban et al. 2014), invertebrados depredadores de semillas (p.ej. Rodríguez et al. 2014), y microorganismos (p.ej. Peris et al. 2015). Los seis trabajos que comprende este monográfico repasan aspectos fundamentales de las interacciones entre plantas y mamíferos frugívoros tales como la importancia de los mamíferos silvestres y domésticos en la colonización de hábitats naturales y humanizados, la complejidad de las interacciones planta-frugívoro, o el papel de los microorganismos en las interacciones planta-frugívoro. Confiamos en que estos trabajos contribuyan a inspirar nuevas investigaciones sobre los patrones, mecanismos y consecuencias recíprocas de las interacciones planta-mamífero frugívoro en el contexto del ciclo biológico completo de las plantas (p.ej. Wang y Smith 2002, Schupp et al. 2010, Fedriani et al. 2012).

\section{Agradecimientos}

Nuestro sincero agradecimiento hacia todos los autores y revisores por su imprescindible contribución para que este monográfico haya podido ser una realidad. También queremos agradecer a Luis Cayuela sus comentarios a un primer borrador $y$, junto a Carolina Puerta-Piñero y a Leyre Jiménez-Eguizábal, su labor de coordinación editorial. JMF fue financiado por la FCT-Portugal, mediante el proyecto "Old-Field Recolonization in the Mediterranean Europe" (Ref. IF/00728/2013).

\section{Referencias}

Bascompte, J., Jordano, P. 2014. Mutualistic networks. Monographs in Population Biology Series, no. 53. Princeton University Press, Princeton. $216 \mathrm{pp}$.

Bronstein, J.L. 1994. Conditional outcomes of mutualistic interactions. Trends in Ecology and Evolution 9:214-217.

Bronstein, J.L., I. Izhaki, R. Nathan, J.J. Tewksbury, O. Speigel, A. Lotan. 2007. Fleshy-fruited plants and frugivores in desert ecosystems. En: Dennis, A.J., Schupp, E.W., Green, R.J., Westcott, D.W. (eds.). Seed dispersal: theory and its application in a changing world, pp. 148-177. Cambridge University Press, Cambridge, Reino Unido.

Campos, C.M., Velez, S. 2015. Almacenadores y frugívoros oportunistas: el papel de los mamíferos en la dispersión del algarrobo (Prosopis flexuosa DC) en el desierto del Monte, Argentina. Ecosistemas 24(3): 2834. Doi.: 10.7818/ECOS.2015.24-3.05

Cipollini, M.L., Levey, D.J. 1997. Secondary metabolites of fleshy vertebratedispersed fruits: adaptive hypotheses and implications for seed dispersal. The American Naturalist 150: 346-372.
Coley, P. D., Barone, J.A. 1996. Herbivory and plant defenses in tropical forests. Annual review of ecology and systematics 305-335.

Chapman, C.A, Russo, S.E. 2006. Primate seed dispersal: linking behavioral ecology with forest community structure. En: Cambell, C. J., Russo, S.E. (eds), Primates in perspective. Pp: 510-525. Oxford University Press. Oxford, Reino Unido.

Dearing, M.D. 1997. The manipulation of plant secondary compounds by a food hoarding herbivore, the North American pika, Ochotona princeps. Ecology 78, 774-781.

Dearing, M.D., Foley, W.J., McLean, S. 2005. The influence of plant secondary metabolites on the nutritional ecology of herbivorous terrestrial vertebrates. Annual Review of Ecology, Evolution and Systematic 36, 169-189.

Dirzo, R., Young, H.S., Galetti, M., Ceballos, G., Isaac, N.J., Collen, B. 2014. Defaunation in the Anthropocene. Science 345: 401-406.

Effiom, E.O., Birkhofer, K., Smith, H.G., Olsson, O. 2014. Changes of community composition at multiple trophic levels due to hunting in Nigerian tropical forests. Ecography 37: 367-377.

Enders, M.S., Vander Wall, S.B. 2012. Black bears Ursus americanus are effective seed dispersers, with a little help from their friends. Oikos 121(4), 589-596.

Escribano-Ávila, G., Pías, B., Escudero, A., Virgós, E. 2015. Importancia ecológica de los mamíferos frugívoros en la dinámica de regeneración de tierras abandonadas en ambientes mediterráneos. Ecosistemas 24(3): 35-42. Doi.: 10.7818/ECOS.2015.24-3.06

Fedriani, J.M. 1996. Dieta anual del zorro, Vulpes vulpes, en dos hábitats del Parque Nacional de Doñana. Doñana Acta Vertebrata 23: 143-152.

Fedriani, J.M., Boulay, R. 2006. Foraging by fearful frugivores: combined effect of fruit ripening and predation risk. Functional Ecology 20: 10701079 .

Fedriani, J.M., Delibes, M. 2011. Dangerous liaisons disperser the Mediterranean dwarf palm: defensive flesh-pulp role against seed predators. Ecology 92: 304-312.

Fedriani, J.M., Delibes, M. 2013. Pulp feeders alter plant interactions with subsequent animal associates. Journal of Ecology 101: 1581-1588.

Fedriani, J.M., Wiegand, T. 2014. Hierarchical mechanisms of spatially contagious seed dispersal in complex seed-disperser networks. Ecology 95:514-526.

Fedriani, J.M., Fuller, T.K., Sauvajot, R. 2001. Does availability of anthropogenic food enhance densities of omnivorous mammals? An example with coyotes in southern California. Ecography 24: 325-311.

Fedriani, J.M., Palomares, F., Delibes, M. 1999. Niche relations among three sympatric Mediterranean carnivores. Oecologia 121: 138-148.

Fedriani, J.M., Zywiec, M., Delibes, M. 2012. Thieves or mutualists? Pulp feeders enhance local endozoochore recruitment. Ecology 93:575-587.

Fedriani, J.M., Wiegand, T., Palomares, F., Grimm, V. 2015. Old-field recolonization under selective defaunation: a spatially-explicit individualbased simulation model. $6^{\text {th }}$ International Simposium-Workshop On Frugivory And Seed Dispersal, Drakensberg, South Africa, 21-26 Junio 2015. pp. 30.

Fleming, T.H., Kress, W.J. 2013. The Ornaments of Life: Coevolution and Conservation in the Tropics. University of Chicago Press, Chicago, Estados Unidos. $616 \mathrm{pp}$.

Fragoso, J.M.V. 1997. Tapir-generated seed shadows: scale-dependent patchiness in the Amazon rain forest. Journal of Ecology 85: 519529.

Freeland, W.J., Janzen, D.H. 1974. Strategies in herbivory by mammals: the role of plant secondary compounds. The American Naturalist 269289.

García, D., Obeso, J.R., Martinez, I. 2005. Spatial concordance between seed rain and seedling establishment in bird-dispersed trees: does scale matter? Journal of Ecology 93: 693-704.

González-Varo, J.P., Fedriani, J.M., JGuitián, J., López-Bao, J.V., SuárezEsteban, A. 2015. Frugivoría y dispersión de semillas por mamíferos carnívoros: rasgos funcionales. Ecosistemas 24(3): 43-50. Doi.: 10.7818/ECOS.2015.24-3.07

Herrera, C.M. 2002. Seed dispersal by vertebrates. En: Herrera, C.M., and Pellmyr, O. (eds.)., Plant-animal interactions: an evolutionary perspective, pp. 186-208. Blackwell, Oxford, Reino Unido.

Herrera, C.M., Jordano, P., Lopez-Soria, L., Amat, J.A. 1994. Recruitment of a mast-fruiting, bird-dispersed tree: bridging frugivore activity and seedling establishment. Ecological Monographs 64: 315-344. 
Holland, J.N., DeAngelis, D.L. 2010. A consumer-resource approach to the density-dependent population dynamics of mutualism. Ecology 91: 1286-1295.

Howe, H.F. 1979. Fear and frugivory. The American Naturalist 114: 925931.

Jordano, P., Schupp, E.W. 2000. Seed disperser effectiveness: the quantity component and patterns of seed rain for Prunus mahaleb. Ecological Monographs 70: 591-615.

Levey, D.J., Silva, W.R., Galetti, M. 2002. Seed Dispersal and Frugivory. CABI, Wallingford, Reino Unido.

Mancilla-Leytón, J.M., Fernández-Ales, R., Martín, A. 2015. Efectividad cualitativa del ganado caprino en la dispersión de especies de frutos secos y carnosos. Ecosistemas 24(3): 22-27. Doi.: 10.7818/ECOS.2015.243.04

O'Reilly-Wapstra, J.M., McArthur, C., Potts, B.M. 2004. Linking plant genotype, plant defensive chemistry and mammal browsing in a Eucalyptus species. Functional Ecology 18(5), 677-684.

Padrón, B., Nogales, M., Traveset, A., Vila, M., Martínez-Abraín, A., Padilla, D.P., Marrero, P. 2011. Integration of invasive Opuntia spp. by native and alien seed dispersers in the Mediterranean area and the Canary Islands. Biological Invasions 13: 831-844.

Perea, R., Delibes, M., Polko, M., Suárez, A., Fedriani, J.M. 2013. Contextdependent fruit-frugivore interactions: partner identities and spatio-temporal variations. Oikos 122: 943-951.

Pérez-Ramos, I.M., Villar, R., Marañón, T. (eds.). 2014. Ecología y gestión de las especies de Quercus. Ecosistemas 23: 1-129.

Peris, J.E., Fedriani, J.M., Peña, L. 2015. Los mamíferos frugívoros prefieren frutos de cítricos infectados por Penicillium: ¿se equivocaba Janzen? Ecosistemas 24(3): 5-13. Doi.: 10.7818/ECOS.2015.24-3.02.

Raguso, R.A. 2008. Wake up and smell the roses: the ecology and evolution of floral scent. Annual Review of Ecology, Evolution, and Systematics 549-569.

Ramos, M.E., González, J.L., Robles, A.B. 2015. Dispersión endozoócora de leguminosas silvestres: desde la recuperación hasta el establecimiento en campo. Ecosistemas 24(3): 14-21. Doi.: 10.7818/ECOS.2015.24-3.03.

Rey, P.J., Alcantara, J. M. 2000. Recruitment dynamics of a fleshy-fruited plant (Olea europaea): connecting patterns of seed dispersal to seedling establishment. Journal of Ecology 88(4), 622-633.
Rodríguez, M., Delibes, M., Fedriani, J.M. 2014. Hierarchical levels of seed predation variation by introduced beetles on an endemic Mediterranean palm. PLOS One. DOI: 10.1371/journal.pone.0109867

Rodríguez, A., Alquezar, B. Peña, L. 2013. Fruit aromas in mature fleshy fruits as signals of readiness for predation and seed dispersal. New Phytologist 197: 36-48.

Rosalino, L.M., Santos-Reis, M. 2009. Fruit consumption by carnivores in Mediterranean Europe. Mammal Review 39(1), 67-78.

Samuni-Blank, M., Izhaki, I., Dearing, M.D., Karasov, W.H., Gerchman, Y., Kohl, K.D., Lymberakis, P., Kurnath, P., Arad, Z. 2013. Physiological and behavioural effects of fruit toxins on seed-predating versus seed-dispersing congeneric rodents. The Journal of experimental biology 216 : 3667-3673

Schaefer, H.M., Ruxton, G. 2011. Plant-Animal Communication. Oxford University, Oxford, United Kingdom.

Schupp, E.W., Jordano, P., Gómez, J.M. 2010. Seed dispersal effectiveness revisited: a conceptual review. New Phytologist 188:333-353.

Suárez-Esteban, A., Delibes, M., Fedriani, J.M. 2013a. Barriers or corridors? The overlooked role of unpaved roads in endozoochorous seed dispersal. Journal of Applied Ecology 50: 767-774.

Suárez-Esteban, A., Delibes, M., Fedriani, J.M. 2013b. Unpaved road verges as hotspots of fleshy-fruited shrub recruitment and establishment. Biological Conservation 167: 50-56.

Suárez-Esteban, A., Delibes, M., Fedriani, J.M. 2014. Unpaved roads disrupt the effect of herbivores and pollinators on the reproduction of a dominant shrub. Basic and Applied Ecology. DOI: 10.1016/j.baae.2014.08.001

Terborgh, J. 2010. Trophic cascades: predators, prey, and the changing dynamics of nature. Island Press, Washington, Estados Unidos.

Traveset, A., Willson, M.F. 1997. Effect of birds and bears on seed germination of fleshy-fruited plants in temperate rainforests of southeast Alaska. Oikos 89-95.

Wang, B.C., Smith, T.B. 2002. Closing the seed dispersal loop. Trends in Ecology and Evolution 17: 379-386.

Whitehead, S.R., Quesada, M.F.O., Bowers, M.D. 2015. Chemical tradeoffs in seed dispersal: defensive metabolites in fruits deter consumption by mutualist bats. Oikos. DOI: 10.1111/oik.02210

Willson, M.F. 1993. Mammals as seed-dispersal mutualists in North America. Oikos 159-176. 